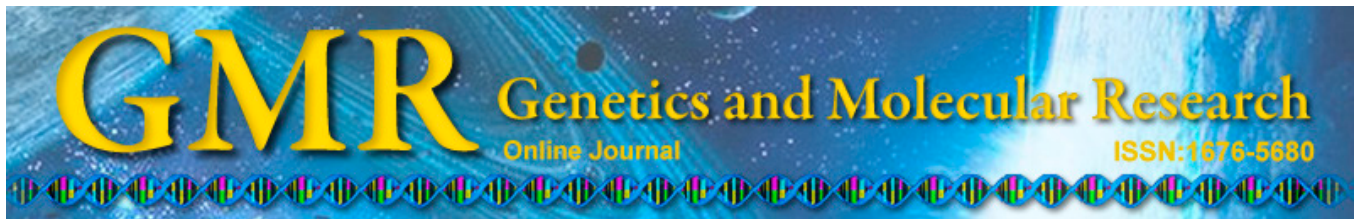

\title{
Correlations among copeptin, ischemia-modified albumin, and the extent of myocardial injury in patients with acute carbon monoxide poisoning
}

\author{
J. Li, J.S. Wang, Z.X. Xie, W.Z. Wang, L. Wang, G.Y. Ma, Y.Q. Li and \\ P. Wang \\ Department of Emergency, Harrison International Peace Hospital, \\ Hengshui, China \\ Corresponding author: J. Li \\ E-mail: lijingvip2014@126.com
}

Genet. Mol. Res. 14 (3): 10384-10389 (2015)

Received January 26, 2015

Accepted May 24, 2015

Published September 1, 2015

DOI http://dx.doi.org/10.4238/2015.September.1.5

\begin{abstract}
This study evaluated the relationships among copeptin, ischemia-modified albumin (IMA), and extent of myocardial injury in patients with acute carbon monoxide poisoning (ACOP). A total of 110 patients with different degrees of ACOP were selected as the poisoning group, and 30 healthy individuals as the control group. The levels of troponin I (cTnI), IMA, and copeptin were detected. Based on the presence of complications, the patients were assigned to the complication (26 patients) or non-complication (84 patients) group. Levels of cTnI, IMA, and copeptin were compared among the control, complication, and non-complication groups. Compared with the control group, in the $2 \mathrm{~h}$ after admission, the IMA levels decreased and copeptin levels increased in the poisoning group; these changes were more significant in patients with severe ACOP than in those with mild ACOP, and the difference was statistically significant $(\mathrm{P}<0.05)$. There were no differences in the IMA and copeptin levels between the groups 7 days after admission; the cTnI levels increased more significantly in patients with severe ACOP than in patients with mild and moderate
\end{abstract}


ACOP, and the differences were statistically significant $(\mathrm{P}<0.05)$. In the complication group, at 7 days after admission, the IMA levels decreased whereas the copeptin and cTnI levels were significantly higher than in the non-complication group, with a statistically significant difference $(\mathrm{P}<0.05)$. IMA was negatively correlated with copeptin. IMA and copeptin detection is clinically useful in the early diagnosis and prognosis of ACOP-related myocardial injury and in guiding early clinical drug application.

Key words: Acute carbon monoxide poisoning; Copeptin; Ischemiamodified albumin; Prognosis

\section{INTRODUCTION}

Acute carbon monoxide poisoning (ACOP) is a common clinical emergency in which the heart is one of the most commonly affected organs, leading to acute myocardial injury followed by disease progression and sudden death. Copeptin is the precursor of arginine vasopressin (AVP), with a similar trend as that of AVP in plasma. In a previous study, it was found to be significantly higher in acute myocardial ischemia patients (Zhang, 2012). Ischemiamodified albumin (IMA) has been shown to increase within minutes of myocardial ischemia (Ma and Li, 2013), which was detected before irreversible damage had occurred. This study was designed to study the relationships among copeptin, IMA, and the extent of myocardial injury in patients with ACOP.

\section{MATERIAL AND METHODS}

\section{Subjects}

A total of 110 ACOP patients [58 men, 52 women; age, 18-74 (mean age, $43.2 \pm$ 13.7) years] who were diagnosed and classified based on the "Occupational ACOP Diagnostic Criteria" by the Ministry of Health"were selected from our hospital emergency department during May 2011 to May 2013 (Wang, 2010). The patients in the poisoning group were further classified into mild poisoning (22 patients), moderate poisoning (50 patients), and severe poisoning (38 patients) groups. Thirty healthy individuals [16 men, 14 women; age, 27-74 (mean age, $45.6 \pm 12.5$ ) years] were selected as the control group. Based on the presence or absence of complications, the patients were divided into complication (26 patients) and noncomplication (84 patients) groups. Patients with a history of severe heart and lung diseases, acute and chronic infectious diseases, sepsis, cardiovascular and cerebrovascular diseases, diabetes insipidus, and other metabolic diseases were excluded. There were no differences in age, gender, and weight among the groups.

\section{Methods and indicators}

Venous blood $(3 \mathrm{~mL})$ was collected intermittently from all patients within $2 \mathrm{~h}(4 \mathrm{~h}$ after poisoning) to 7 days after admission, and troponin I (cTnI 0-1.7 ng/mL) and IMA (IMA 
$>65 \mathrm{U} / \mathrm{mL}$ ) levels were detected using the 7600 automatic biochemical analyzer by Hitachi (Tokyo, Janpan). Another sample of blood collected $(3 \mathrm{~mL}$ ) was centrifuged at $3000 \mathrm{r} / \mathrm{min}$ for $10 \mathrm{~min}$ and separated. The plasma specimens were stored at $-80^{\circ} \mathrm{C}$ for detecting the copeptin level. Copeptin was detected using ELISA test assay, and the kit was provided by Phoenix Pharmaceuticals (San Francisco, CA, USA).

\section{Statistical analysis}

Statistical analysis was carried out using the SPSS 16.0 software (Chicago, IL, USA). Measurement data of the two groups were compared using the $t$-test, and that of multiple groups were compared using variance analysis; count data were compared using the chisquare test; and correlation analysis was carried out using the Pearson correlation test. $\mathrm{P}<$ 0.05 was considered to be statistically significant.

\section{RESULTS}

\section{Comparison of IMA, copeptin, and cTnI in patients with different degrees of ACOP}

Compared with the control group, within $2 \mathrm{~h}$ after admission, the IMA level of the poisoning group decreased and the copeptin level increased in the poisoning group, with severe ACOP patients showing a more significant increase than those with mild ACOP, and the difference was statistically significant $(\mathrm{P}<0.05)$. No statistically significant difference existed in the levels of cTnI between the two groups. There was no significant difference in the levels of IMA and copeptin between the groups 7 days after admission. However, the cTnI concentration increased more significantly in patients with severe ACOP than in those with mild and moderate ACOP, with statistically significant differences $(\mathrm{P}<0.05)$ (Table 1$)$.

\begin{tabular}{|c|c|c|c|c|c|c|c|c|}
\hline \multirow[t]{2}{*}{ Group } & \multicolumn{2}{|c|}{ Control (30) } & \multicolumn{2}{|c|}{ Mild (22) } & \multicolumn{2}{|c|}{ Moderate (50) } & \multicolumn{2}{|c|}{ Severe (38) } \\
\hline & $2 \mathrm{~h}$ & 7 days & $2 \mathrm{~h}$ & 7 days & $2 \mathrm{~h}$ & 7 days & $2 \mathrm{~h}$ & 7 days \\
\hline $\mathrm{cTnI}(\mathrm{ng} / \mathrm{mL})$ & $1.27 \pm 0.28$ & $1.31 \pm 0.3$ & $1.33 \pm 0.23$ & $1.8 \pm 0.17^{\#}$ & $1.26 \pm 0.27$ & $2.34 \pm 0.46^{\# \Delta}$ & $1.22 \pm 0.41$ & $2.66 \pm 0.54^{\# \Delta *}$ \\
\hline $\operatorname{IMA}(>65 \mathrm{U} / \mathrm{mL})$ & $67.23 \pm 1.4$ & $67.93 \pm 1.34$ & $62.50 \pm 2.17^{\#}$ & $67.95 \pm 1.25$ & $59.04 \pm 3.1^{\# \Delta}$ & $66.5 \pm 1.63$ & $56.01 \pm 8.85^{\# \Delta *}$ & $65.51 \pm 10.08$ \\
\hline Copeptin $(\mathrm{ng} / \mathrm{mL})$ & $0.87 \pm 0.19$ & $0.86 \pm 0.17$ & $2.82 \pm 0.73^{\#}$ & $2.39 \pm 0.37$ & $7.31 \pm 0.95^{\# \Delta}$ & $2.52 \pm 0.58$ & $13.08 \pm 1.96^{\# \Delta *}$ & $2.65 \pm 0.57$ \\
\hline
\end{tabular}

Compared with the control group, ${ }^{\#} \mathrm{P}<0.05$; compared with the mild group, ${ }^{\Delta} \mathrm{P}<0.05$, compared with the moderate group, ${ }^{*} \mathrm{P}<0.05$,

\section{Comparison of IMA, copeptin, and cTnI between complication and non-complication groups}

There were 26 patients with complications, which accounted for $24 \%$ of the total number. Compared with the non-complication group, the IMA level of the complication group decreased, and the copeptin level increased. The cTnI levels of the complication group, at 7 days after admission, were significantly higher than those of the non-complication group. The difference was statistically significant $(\mathrm{P}<0.05)$ (Table 2$)$. 
Table 2. Comparison of cTnI, IMA, and copeptin between complication and non-complication groups (means \pm SD).

\begin{tabular}{|c|c|c|c|c|}
\hline \multirow[t]{2}{*}{ Group } & \multicolumn{2}{|c|}{ Complication (26) } & \multicolumn{2}{|c|}{ Non-complication (84) } \\
\hline & $2 \mathrm{~h}$ & 7 days & $2 \mathrm{~h}$ & 7 days \\
\hline $\mathrm{cTnI}(\mathrm{ng} / \mathrm{mL})$ & $1.27 \pm 0.28$ & $3.10 \pm 0.22^{\Delta}$ & $1.30 \pm 0.27$ & $1.87 \pm 0.27$ \\
\hline $\mathrm{IMA}(>65 \mathrm{U} / \mathrm{mL})$ & $54.62 \pm 1.53^{\Delta}$ & $60.65 \pm 3.61^{\Delta}$ & $57.89 \pm 4.02$ & $66.84 \pm 1.78$ \\
\hline Copeptin $(\mathrm{ng} / \mathrm{mL})$ & $13.88 \pm 1.45^{\Delta}$ & $6.65 \pm 1.82^{\Delta}$ & $6.99 \pm 3.39$ & $2.47 \pm 0.61$ \\
\hline
\end{tabular}

Compared with the non-complication group, ${ }^{\Delta} \mathrm{P}<0.05$.

\section{Correlation between copeptin, IMA, and the extent of myocardial injury}

Myocardial injury in ACOP patients increased with a corresponding increase in the copeptin level and decrease in the IMA level. Correlation analysis showed that during the early stage in patients with different degrees of ACOP, IMA had a negative correlation with copeptin $(\mathrm{r}=-0.560, \mathrm{P}<0.01)$.

\section{DISCUSSION}

ACOP is a common medical emergency with the highest mortality among acute poisoning cases (Chen and Lin, 2009). It primarily causes tissue hypoxia and multiple organ damage, with heart damage such as arrhythmia, heart failure, and myocardial infarction being the most common. This may be due to: 1) increasing cardiac load and increasing oxygen demand caused by stimulation of the adrenergic system; 2) energy deficiency in myocardial cells caused by vascular endothelial damage and damage to the mitochondrial function; 3) oxygen tension reduction in capillaries due to the oxygen dissociation curve lofting and reduced oxygen release from blood to tissues; 4) myocardial cell surface ion channel dysfunction and inability to restore the physiological state due to various ions inside and outside the myocardial cells causing ion distribution imbalance and $\mathrm{Ca}$ overload in the cytoplasm, thereby triggering myocardial cell lysis; 5) possible production of large amounts of oxygen-free radicals, causing intracellular acidosis, inhibition of oxidative phosphorylation, and ATP depletion, which damages myocardial function. As a specific marker of myocardial dysfunction, serum concentration of cTnI was elevated at 4-6 h, reached a peak at 18-24 h, and dropped to normal after 4 weeks, when it was used for diagnosis of ACOP myocardial injury. Therefore, it is particularly important to explore novel sensitive markers for myocardial ischemia and necrosis.

Copeptin, which is secreted by the hypothalamus, consists of a 39 -amino acid sequence of glycoprotein and serves as a new biochemical marker. It correlates with the occurrence, development, and prognosis of many diseases. The functions of copeptin are uncertain; it probably assists the misfolded monomer to refold by calnexin/calreticulin protein systems to ensure the stability of their biological effects. The function of copeptin is unclear; it is likely that based on the calnexin/calreticulin role of protein systems, it assists in auxiliary misfolded monomer refolding in order to ensure the stability of its biological effects. Copeptin correlates with myocardial ischemia (Chai et al., 2009). It increases in 0-4 h and decreases after $6 \mathrm{~h}$. Another previous study also demonstrated (Reichlin et al., 2009) that copeptin was a stress hormone. A disorder of the copeptin level can affect the development of many diseases. It is clinically useful in the early diagnosis, assessment, and prognosis of disease. Because of its relatively high sensitivity but poor specificity, combined IMA detection was used to make up 
for the shortcomings of specificity in the early diagnosis of AOCP-related myocardial injury. IMA is formed by changes in human serum albumin flowing through ischemic tissue. When local tissue ischemia produces oxidative stress, sequence undergoes oxidative modification to form IMA. It reflects a substantive change before cell necrosis, which develops 5-10 min after myocardial ischemia. In a previous study, peripheral blood IMA concentrations increased rapidly and continued to rise in the ischemic process, which lasted 2-4 h, and then returned to baseline level within 6-10 h (Cao et al., 2011). The detection of myocardial ischemia before irreversible damage will be helpful for early prediction and detection. However, IMA did not show a high degree of tissue or clinical specificity in myocardial injury. The specificity increased in non-cardiac myocardial injury, and copeptin was expected to fill the gap.

In this study, dynamic monitoring of serum IMA and copeptin levels in patients with ACOP showed that in the early stage, varying degrees of changes occurred in the concentrations of IMA and copeptin in patients with different degrees of poisoning, and the changes were related with the extent of $\mathrm{CO}$ poisoning. More severe poisoning and more severe heart and other organ damage were found to be associated with lower IMA levels and higher copeptin levels. Due to the short duration, markers of myocardial injury were not increased, which did not reflect the degree of the lesion. At 7 days after admission, the IMA and copeptin levels began to decrease, and there was no difference between the poisoning groups. With the occurrence of ACOP complications, the copeptin level increased significantly. There was a significant difference in the IMA and copeptin levels between the complication and noncomplication groups, suggesting that there may be multiple organ damage in ACOP patients. Because of the disease progression, IMA and copeptin levels in the complication group continued to rise, or rose again after decline, indicating severe myocardial injury and poor prognosis in the patients. Differences in the levels of IMA and copeptin were consistent with the disease outcome in this study. There was a negative correlation between IMA and copeptin in patients with ACOP myocardial injury; the lower the IMA level, the higher was the copeptin level, and the more severe the myocardial injury, the more severe was the tissue hypoxia. The results of this study, to some extent, prompted that IMA and copeptin levels may reflect the extent of ACOP-related myocardial injury and occurrence of complications. Some studies have shown (Kao et al., 2009) the occurrence and severity of myocardial injury after CO poisoning increased with the severity of poisoning, and it was closely related to short-term complications and prognosis. In patients with moderate and severe $\mathrm{CO}$ poisoning, the degree of myocardial injury can predict the incidence of complications, as seen in this study. Most myocardial injury is reversible in ACOP patients, with the standard treatment resuming in a few days. Some indicators continued to rise, or rose again after decline, during the dynamic monitoring process, indicating disease progression and the need of timely adjustments for treatment, which is important in guiding early clinical drug application.

In summary, the higher the levels of IMA and copeptin, the more severe were the ACOP myocardial injury and tissue hypoxia. With increase in the severity of poisoning, a significant increase occurs in cardiac injury, which can be used to assess mortality in patients. Dynamic monitoring of IMA and copeptin levels can be used in the early diagnosis of myocardial injury and as a reference for ACOP prognosis.

\section{Conflicts of interest}

The authors declare no conflict of interest. 


\section{REFERENCES}

Cao H, Yu SB, Qin M, Qin M, et al. (2011). Chest pain within $6 \mathrm{~h}$ ischemia modified albumin research on the value of the prognosis of acute myocardial infarction. ZhongGuo Shi Yong NeiKeZaZhi 8: 629-631.

Chai SB, Hui YM, Li XM, Xiao Y, et al. (2009). Plasma levels of copeptin in patients with coronary heart disease. Heart Vessels 24: 79-83.

Chen BZ and Lin GW (2009). Practical internal medicine. Ren Min Wei Sheng Chu Ban She 1590-1593.

Kao HK, Lien TC, Kou YR and Wang JH (2009). Assessment of myocardial injury in the emergency department independently predicts the short-term poor outcome in patients with severe carbon monoxide poisoning receiving mechanical ventilation and hyperbaric oxygen therapy. Pulm. Pharmacol. Ther. 22: 473-477.

Ma CH and Li LX (2013). With myocardial ischemia modified albumin type fatty acid binding protein value in early diagnosis of acute myocardial infarction is reviewed. ZhongHua Shi Yong Zhen DuanyuZhi Liao ZaZhi 2: 107-109.

Reichlin T, Hochholzer W, Stelzig C, Laule K, et al. (2009). Incremental value of copeptin for rapid rule out of acute myocardial infarction. J. Am. Coll. Cardiol. 54: 60-68.

Wang TX (2010). High doses of vitamin C for medium and severe myocardial protection in patients with acute carbon monoxide poisoning. ZhongGuo Yi Shi Jin XiuZaZhi 25: 46-48.

Zhang SL (2012). The clinical value of plasma brain natriuretic peptide and peptide hormone levels and the extent of coronary artery disease in patients with coronary heart judgment. ZhongGuo Yi Shi Jin XiuZaZhi 28: 63-64. 